\title{
La calidad del servicio al cliente y la satisfacción laboral en la industria hotelera de las ciudades de Panamá y de Colón
}

\author{
Miroslava Alzamora de Zárate ${ }^{1, *}$ \\ ${ }^{1}$ Universidad Católica Santa María la Antigua, Facultad de Negocios, República de Panamá.
}

*Autor para correspondencia. E-mail: malzamora@usma.ac.pa

Recibido: 29 de septiembre de 2020

Aceptado: 12 de octubre de 2020

\begin{abstract}
Resumen
Panamá por ser un sitio turístico y contar con una economía de servicio, la industria hotelera juega un papel muy importante. Según estadísticas señaladas, el turismo, junto a los servicios y actividades relacionadas, integra uno de los sectores más vigorosos en la economía. La industria hotelera representa unos 3 mil millones de dólares de inversión turística. y la industria genera alrededor de 40 mil empleos, pero en los últimos dos años se han perdido más de 25 mil puestos de trabajo, a lo que se sumará el efecto del alza del salario mínimo, que a su juicio afectará aún más a la actividad.Es por ello que el objetivo de este estudioes analizar la calidad del servicio al cliente y la satisfacción laboral en la industria hotelera de la ciudad de Panamá y de la ciudad de Colón.El tipo de investigación es descriptiva de diseño no experimental, y de campo; para recoger los datos se utilizaron dos cuestionario: uno se le aplicó a 445 clientes y el otro a 484 colaboradores para reunir la información, y luego se presentaron los resultados a través de cuadros con estadísticas descriptivas. La investigación desarrollada concluyó entre otros aspectos, en que los hoteles en Panamá cuentan con política o procedimiento en la busca de la satisfacción del cliente, pero la frecuencia de capacitación no es la adecuada. Es necesario divulgar y capacitar a todo el personal que el servicio al cliente se ha convertido en motivo esencial en la decisión del cliente al escoger donde realizar la compra de algún producto y/o servicio en especial en las empresas en las cuales su naturaleza es la prestación de servicio, como la industria hotelera, todo esto da como resultado que el servicio actualmente sea un factor fundamental en la competitividad en los negocios.
\end{abstract}

Palabras Clave: Calidad, Servicio al cliente, Satisfacción Laboral, Industria hotelera, Turismo 


\begin{abstract}
Panama, being a tourist site and having a service economy, the hotel industry plays a very important role. According to indicated statistics, tourism, along with related services and activities, is one of the most vigorous sectors in the economy. The hotel industry represents about 3 billion dollars of tourism investment. and the industry generates around 40 thousand jobs, but in the last two years more than 25 thousand jobs have been lost, to which will be added the effect of the increase in the minimum wage, which in his opinion will affect the activity even more. For this reason, the objective of this study is to analyze the quality of customer service and job satisfaction in the hotel industry of Panama City and Colon. The type of research is descriptive of non-experimental design, and of countryside; to collect the data, two questionnaires were used: one was applied to 445 customers and the other to 484 collaborators to gather the information, and then the results were presented through tables with descriptive statistics. The investigation carried out concluded, among other aspects, that the hotels in Panama have a policy or procedure in order to achieve customer satisfaction, but the frequency of training is not adequate. It is necessary to disclose and train all staff that customer service has become an essential reason in the customer's decision when choosing where to purchase a product and / or service, especially in companies in which its nature is the service provision, like the hotel industry, all this results in service being a fundamental factor in business competitiveness today.
\end{abstract}

Keywords: Quality, Customer Service, Labor Satisfaction, Hotel Industry, Tourism

\title{
1. Antecedentes
}

El turismo es una de las actividades económicas más importantes de Panamá y se estima que los ingresos de este sector duplican a los generados por el Canal de Panamá alcanzando cifras superiores a los B/ 4,451.400.000 millones de balboas en el 2017.

En el servicio turístico, principalmente en la industria hotelera, la calidad es de suma importancia; ya que ante estos días de agresiva competencia y una sobre oferta de habitaciones que cada día crece más y más, los clientes se vuelven más exigentes. Esto implica una serie de expectativas personales que el turista desea cumplir cuando visita un país y se hospeda en un hotel. Es por ello que cuando se habla de Calidad, se refiere a satisfacer las necesidades de los clientes y las expectativas de estos.

La falta de conocimientos del tema servicio al cliente y su aplicación en los hoteles y la importancia de satisfacer también a sus clientes internos, son solamente obstáculos que no permite a las empresas hoteleras mejorar su posicionamiento y su rentabilidad.

Como parte de la necesidad de mejorar esta problemática, es que se realiza la siguiente investigación, la cual analiza la calidad del servicio al cliente y la satisfacción laboral en la industria hotelera en la ciudad de Panamá y la ciudad de Colon.

Puesto que la productividad es una variable clave para garantizar la supervivencia de cualquier empresa a largo plazo, la identificación de aquellos factores relevantes para incrementar la productividad en este primer eslabón de la cadena resulta de vital importancia para el futuro del sector y de parte importante de la economía andaluza. Sin embargo, el estudio de esta relación no es una tarea fácil. En primer lugar, la definición y medición de la productividad da lugar a una serie de 
problemas cuando tratamos de utilizarla para las actividades de servicios, en general, y la actividad hotelera, en particular. En segundo lugar, el concepto de calidad está sujeto a muy diversas interpretaciones y enfoques, problema que se acentúa en el caso de los servicios.

La escasa existencia de estudios que evalúen de forma explícita la relación entre calidad y productividad en el sector hotelero justifica el interés de conocer de forma empírica de qué manera puede afectar una variable sobre la otra. Una de las recomendaciones que brinda este trabajo es que, entre los distintos modelos de gestión de la calidad total, el Modelo EFQM de Excelencia es especialmente idóneo por su carácter práctico. Está integrado por unos conceptos fundamentales de la excelencia, nueve elementos a través de los que las empresas pueden evaluar el logro de dicha excelencia y un esquema de gestión y evaluación. Dada su relevancia, este modelo es la base del análisis de la incidencia de la calidad sobre la productividad, objeto de este trabajo.

Ulacia (2015), en su tesis doctoral titulada, Factores que inciden en la calidad de la experiencia alojativa en hoteles de Ciudad 5 y 6 estrellas en la Habana, comentan que los sistemas de gestión de la calidad que se implementan en la mayoría de las instalaciones hoteleras, en particular las cubanas, despliegan procesos de medición y evaluación de la calidad del servicio donde predominan los aspectos internos gestionados y controlados por los hoteles.

Analizando el trabajo con responsables de hoteles de 4 y 5 estrellas de La Habana, delimitamos los principales factores para la medición y evaluación de la calidad del servicio. Los resultados muestran que los responsables centran su atención, principalmente, en componentes de tipo interno y relegan a un segundo término componentes externos, pese al impacto directo de éstos sobre la percepción de calidad de la experiencia alojativa.

El análisis de narraciones de las experiencias vividas por clientes de los hoteles colaboradores, mediante Tripadvisor, refleja la importancia para los clientes de los aspectos de tipo externo. El ámbito externo Ubicación-entorno registró la mayor parte de elementos indicados. En este ámbito predominan los elementos asociados a experiencias positivas respecto a las negativas (9.34 veces más), igual que en el ámbito Amabilidad (10.33 veces más de elementos positivos). La mayor proporción de elementos asociados a experiencias negativas se da en los ámbitos de Internet y tecnologías de comunicación.

\section{Justificación de la Investigación}

El presente estudio profundiza no solamente en el diagnóstico que realiza acerca de la problemática general de la calidad del servicio al cliente y la forma como ésta afecta a una de las principales áreas de la industria panameña, sino también en la satisfacción laboral de los colaboradores que brindan sus servicios en la industria hotelera de la ciudad de Panamá y de la ciudad de Colón.

Existen muy pocas investigaciones en nuestro país que relacionen la satisfacción laboral con la calidad del servicio al cliente. En efecto, la mayor parte de los estudios sobre satisfacción laboral se han orientado a relacionarla con variables tales como rendimiento laboral, motivación laboral, estilos de supervisión, reconocimiento, etc.

Este estudio asume que la satisfacción laboral repercutirá sobre la calidad del servicio que se prestará al cliente, lo que en última instancia deberá traducirse en satisfacción del cliente y, consecuentemente en estabilidad y crecimiento de este importante sector de la industria panameña. 
Definir la calidad del servicio al cliente y determinar cómo evaluarlos ha sido motivo de grandes divergencias y profundos estudios entre diversos autores; el presente trabajo, se ha apoyado en primer lugar, de una revisión bibliográfica respecto a algunos de los modelos de evaluación más representativos en la literatura científica y de otras fuentes de importancia que hemos consultado donde se presenta un compendio de referencias y de información con el objetivo de que sirva de base para futuros trabajos de igual transcendencia, así como para la toma de decisiones estratégicas en la industria hotelera.

Entre otras bases de datos consultas se encuentran las veintitrés bases de datos puestas a disposición del usuario por Pro Quest para una consulta simultánea, la mayoría en el área ciencias sociales y economía. También se consultaron algunas tesis doctorales que han investigado sobre el tema en diferentes países. Algunas de las fuentes de mayor relevancia que se han consultado para el estudio de los temas de esta investigación son: Asociación Panameña de Hoteles, Autoridad de Turismo de Panamá, Cámara Panameña de Turismo, Contraloría General de la República de Panamá.

En segundo lugar, se ha tomado en consideración diversas perspectivas teóricas y de investigación que caracterizan el trabajo de algunos autores que han tratado las variables en las que se fundamenta este trabajo. A partir de los cuales se han diseñado diversos modelos de evaluación considerando para ello los atributos valorados para la satisfacción laboral y para la prestación de un servicio excelente.

Por otra parte, el tema de la satisfacción laboral ha sido conceptualizado de múltiples maneras dependiendo de las posiciones teóricas asumidas y manejadas por los diferentes autores. Estas diferentes posiciones teóricas, evidencian que la satisfacción laboral es un fenómeno en el que influyen múltiples variables.

Para los fines de este estudio se utilizó como instrumento de medición de la misma, el cuestionario desarrollado por Coexphal, Euroempleo y cofinanciado por el Servicio Andaluz de Empleo y el Fondo Social Europeo, en el marco del Programa para la cooperación transnacional e interregional en el ámbito del empleo.

Con este cuestionario se obtiene información de los trabajadores relativa a su compromiso con la empresa u organización, valoración relacionada con las condiciones de trabajo relativas a la seguridad, higiene y ergonomía, e información relacionada con los planes de formación continua organizada por la empresa con la finalidad de mejorar las condiciones de trabajo.

Algunos autores indican que, las características personales juegan el papel decisivo en la determinación de los niveles individuales de satisfacción. El ser humano es único e irrepetible, por lo tanto, sus niveles de satisfacción laboral serán también específicos a cada persona.

Los niveles de satisfacción estarán condicionados por lo que la persona haya vivido, mejor dicho, por su historia, la edad, el sexo, las aptitudes, la autoestima, la autovaloración y el entorno sociocultural donde se desenvuelve. Estas particularidades desarrollarán un conjunto de expectativas, necesidades y aspiraciones en relación a las áreas personal y laboral que determinarán los niveles antes mencionado. 
La importancia teórica de este estudio radica en analizar las diversas perspectivas que caracterizan la postura de algunos autores para definir calidad del servicio y la satisfacción laboral, y a partir de los cuales se han diseñado diversos modelos de evaluación considerando para ello, los atributos valorados en la prestación de un servicio excelente. Estos están determinados por las perspectivas desde las cuales se formulan, siendo dos las encontradas en la literatura, a saber: la perspectiva expectativas-percepciones y la perspectiva objetiva-subjetiva (también llamada Interno-Externo).

Es por ello que, en lo teórico, el presente estudio adquiere relevancia en la medida que desarrolla un aspecto de la ciencia, al explicar la importancia del servicio al cliente y la satisfacción laboral para lograr alcanzar los mejores resultados dentro de las empresas hoteleras. Considerando que en muchas ocasiones esta condición no es tomada en cuenta a fin de lograr la satisfacción de sus colaboradores quienes a su vez se identifican con el ofrecimiento de un servicio de calidad a los clientes.

En la práctica, la calidad del servicio al cliente se ha convertido en una estrategia empresarial. Es utilizada como herramienta de ventas por lo que es un factor muy importante ya que el satisfacer a un cliente o bien, exceder sus expectativas puede fidelizarlo, quien, a su vez, puede transmitir su satisfacción a otros potenciales clientes, lo que trae consigo grandes beneficios para el sector hotelero. El brindar una excelente atención debe ser permanente y de variar, debe ser para exceder las expectativas de los clientes.

El sector servicios se caracteriza por un contacto intensivo con los clientes. Por tanto, su satisfacción constituye un componente de esencial importancia en la línea de éxito de las organizaciones que pertenecen a este sector. En esta misma dirección, autores como Spinelli y Canavos (2000) sugieren que una de las fuentes para conseguir la fidelización del cliente es el mantenimiento de empleados satisfechos por parte de la empresa. Ello implica que los directivos de establecimientos hoteleros desean encontrar a un tipo de empleado cuya personalidad, motivación y habilidades contribuyan a la satisfacción del cliente y al cumplimiento de los objetivos de la organización.

Por lo que este estudio brindará valiosa información que permitirá a los administradores y gerentes de atención a clientes de los hoteles de ciudad de Panamá realizar un mejor diagnóstico para el diseño e implementación de estrategias que permitan la mejora continua de las dimensiones más relevantes de la calidad de servicio acorde con su realidad.

En lo metodológico, esta investigación implica el uso de ciertos métodos, técnicas, procedimientos e instrumentos que están a disposición de otros investigadores que se interesen por investigar estas variables en otros contextos y realidades. El estudio plantea un método de trabajo para investigar la relación entre la satisfacción laboral y la calidad del servicio al cliente y podrá ser utilizado en otros trabajos de investigación.

La investigación permitirá conocer la satisfacción laboral y el desempeño del colaborador. Los resultados contribuirán a la optimización de la gestión empresarial, apostando por la generación de un ambiente favorable en el cual los colaboradores se desempeñen a satisfacción y que les brinden una atención personalizada a los clientes. 


\section{Materiales y métodos}

En la presente investigación se seleccionó el tipo de investigación descriptiva, debido a que las dos variables en estudio (la calidad del servicio al cliente y la satisfacción laboral) se medirán de manera independiente para determinar cómo son y cómo se manifiestan en el sector de la industria hotelera de la ciudad de Panamá y de la ciudad de Colón y no como se relacionan dichas variables.

Debido a que los datos son recolectados directamente en el sitio donde se encuentra el objeto de estudio podemos decir que la presente investigación tiene un diseño de campo. Por lo tanto, en este estudio no se construye ninguna situación, sino que se observan situaciones existentes, no provocadas intencionalmente, de modo que el diseño de la investigación parte de la observación u posterior interpretación y análisis de resultados.

Dentro de la investigación no experimental, se seleccionó la transeccional descriptiva, ya que se recolecta la información, para describir el estado de las variables, en su ambiente natural, en un solo momento y en un momento dado.

Para determinar la población es necesario señalar que esta investigación se realiza en los hoteles de Ciudad de Panamá y Colón. Según datos proporcionados por la Autoridad de Turismo de Panamá, son 89 hoteles en Ciudad de Panamá y 14 hoteles en Ciudad de Colón. Se visitaron todos ellos. De todos ellos, solo 20 hoteles de la Ciudad de Panamá aceptaron participar en la investigación, y de Colón sólo 3 decidieron participar.

Las unidades de análisis están compuestas por dos grupos de personas dentro de estos hoteles específicos, que son:

- Los clientes o huéspedes de los hoteles

- $\quad$ Los colaboradores de hoteles

En cuanto a los colaboradores, se totalizó la cantidad de personal por hoteles, lo cual es de 1567 para Ciudad de Panamá (20 hoteles) y de 315 en Ciudad de Colón (3 hoteles), para un total de 1882 colaboradores.

Para determinar a los clientes, los hoteles proporcionaron un promedio diario. Para Ciudad de Panamá fue de 1351 (20 hoteles) y de 230 en Ciudad de Colón (3 hoteles), para un total de 1581 clientes.

Como se puede observar en el cuadro anterior, para el caso de los colaboradores el total de la población es de 1,882 personas, y de los clientes o huéspedes es de 1,581 personas.

Para el caso de los colaboradores se tiene una confiabilidad de $95 \%$, y probabilidad de éxito (p) conservadora de $.5, \mathrm{q}=.5$, con un error de $5 \%$, y población $(\mathrm{N})$ de 1882 colaboradores de Hoteles. $\mathrm{Al}$ calcular Z en la tabla estadística para cálculos de Z, nos da un valor de 1.96. Ahora se procede a reemplazar en la fórmula, y nos da la siguiente respuesta:

En relación a los instrumentos, se utilizaron dos cuestionarios: El primero se permitirá obtener los datos para determinar la calidad de servicio en los clientes o los huéspedes, está tomado en el modelo ServiceQuality (SERVQUAL), el cual es considerado por los especialistas un referente 
importante, siendo citado, analizado y aplicado en numerosas y recientes investigaciones sobre diversos tipos de servicios. Es por esta razón que será la base de la medición que se realizará en varios hoteles ubicados en la ciudad de Panamá. Como ya se ha explicado no fue necesario construir un instrumento ya que el utilizado ya ha sido probado en muchas investigaciones.

La validez de un instrumento se refiere al grado en que el instrumento mide aquello que pretende medir y la confiabilidad es de la consistencia interna del instrumento, que se puede estimar con el alfa de Cronbach.

El primer instrumento aplicado a los clientes o huéspedes de los hoteles está basado en el modelo SERVQUAL, es un cuestionario con preguntas estandarizadas para la Medición de la Calidad del Servicio, herramienta elaborada por Zeithaml, Parasuraman y Berry (1990) y desarrollado en los Estados Unidos con el auspicio del Marketing Science Institute, fue validado para América Latina por Michelsen Consulting con el apoyo del nuevo Instituto Latinoamericano de Calidad en los Servicios. El Estudio de Validación de este instrumento concluyó en junio de 1992.

Las 22 variables que integran el cuestionario definitivo constituyen una adaptación de SERVQUAL original, realizada tomando como referencia investigaciones anteriores efectuadas en el sector de alojamiento (Getty y Thompson, 1994; Ruiz, Vázquez y Díaz, 1995; Falces et al, 1999), por lo que se puede considerar que las escalas presentan validez de contenido.

La Confiabilidad de este instrumento se determinó a partir del cálculo de coeficiente del alfa de Cronbach. Antes de calcular el alfa de Cronbach se realizó un análisis factorial para identificar el número de variables y el número de ítems que son significativas para cada variable.

\section{Resultados}

A continuación, se exponen, analizan y discuten los datos obtenidos en los instrumentos de investigación que fueron impartidos. Comenzaremos por la información de los clientes y luego la de los colaboradores.

\section{Resultados del cuestionario aplicado a los Clientes}

En cuanto al género, un 58.0\% son del sexo masculino, y un $42.0 \%$ son del sexo femenino. En relación a la edad, un 54.2\% tienen edades entre 36 a 50 años, un 24.5\% tienen edades entre 21 a 35 años. Además, un 15.1\% tienen edades de 51 a 65 años, y un 6.3\% tienen 20 o menos años. Esto indica que los clientes son personas maduras y con edades promedias a los 50 años.

En cuanto al nivel educativo un $82.8 \%$ tienen estudios universitarios, un $7.2 \%$ tienen otros niveles educativos. En cuanto al país de origen, un 34.8\% son del país de Panamá, un 23.1\% son de Colombia, un $11.9 \%$ son de Costa Rica, un 11.9\% son de España, un $9.9 \%$ son de Guatemala, un 4.7\% son de Argentina, y con porcentaje menores a 4\% están otros países de Iberoamérica.

En cuanto al total de visitas a Panamá, se observa tanto en el Cuadro 10, que un 58.2\% dicen que han visitado a Panamá en más de 10 veces, esto es así por motivo de negocios, un 15.5\% han visitado de 5 a 10 veces, un 13.5\% han visitado entre 2 a 4 veces, y un $12.8 \%$ lo han visitado solo una vez. 
A continuación, se presenta la información sobre calidad del servicio recogida a través del cuestionario impartido a los clientes de los hoteles que fueron 445 clientes, las preguntas corresponden a cada dimensión a medir y a los Ítems que se desarrollan por dimensión. Este cuestionario se puede consultar en Anexo A.

\section{Variable Calidad de Servicio al Cliente \\ Dimensión: Elementos tangibles \\ Indicadores: Instalaciones, empleados y Materiales \\ Ítems: $1,2,3,4$ y 5}

Esta dimensión evalúa las instalaciones, empleados y los materiales con que cuenta el hotel. Los elementos tangibles se evaluaron con 5 preguntas, la cuales se presentan en el cuadro siguiente, y con los cálculos de su media y desviación interna por pregunta.

\section{Cuadro $\mathbf{N}^{\circ} 1$}

\section{Elementos tangibles}

\begin{tabular}{|c|c|c|}
\hline & $\mathrm{N}$ & Media \\
\hline 1-El hotel cuenta con instalaciones modernas y atractivas & 445 & 3.87 \\
\hline $\begin{array}{l}\text { 2-Los empleados del hotel tienen una apariencia limpia y } \\
\text { agradable }\end{array}$ & 445 & 3.96 \\
\hline $\begin{array}{l}\text { 3-La habitación cuenta con todos los servicios necesarios para } \\
\text { sentirme cómodo durante mi estancia }\end{array}$ & 445 & 3.89 \\
\hline $\begin{array}{l}\text { 4-El hotel cuenta con todas las instalaciones, facilidades y } \\
\text { servicios que necesito para disfrutar de mi estadía }\end{array}$ & 445 & 3.95 \\
\hline $\begin{array}{l}\text { 5-El servicio de alimentos y restaurantes con que cuenta el } \\
\text { hotel ofrecen comida de calidad, con menús variados y } \\
\text { excelente cocina }\end{array}$ & 445 & 3.44 \\
\hline
\end{tabular}

Fuente: Alzamora (2018).

En el área elementos tangibles sobresalen en el acuerdo positivo, mayormente las siguientes afirmaciones:

- Los empleados del hotel tienen una apariencia limpia y agradable

- El hotel cuenta con todas las instalaciones, facilidades y servicios que necesito para disfrutar de mi estadía

- La habitación cuenta con todos los servicios necesarios para sentirme cómodo durante mi estancia 


\section{Dimensión: Empatía \\ Indicadores: Necesidades, Disposición, Atención y Ambiente}

Esta categoría evalúa la atención individualizada que la firma facilita a sus clientes. La Empatía se evalúa con 5 preguntas, la cuales se presentan en el cuadro siguiente, y con los cálculos de sus medias y su desviación estándar por pregunta.

\section{Cuadro $\mathrm{N}^{\circ} 2$ \\ Empatía}

\begin{tabular}{|c|c|c|}
\hline & $\mathrm{N}$ & Media \\
\hline $\begin{array}{l}\text { 6-El personal del hotel siempre está atento a mis deseos y } \\
\text { necesidades }\end{array}$ & 445 & 4.02 \\
\hline $\begin{array}{l}\text { 7-El personal del hotel siempre está con una sonrisa en el } \\
\text { rostro; se muestra interesado por servir a los huéspedes }\end{array}$ & 445 & 3.89 \\
\hline 8-En el hotel me prestan una atención muy personal & 445 & 3.94 \\
\hline $\begin{array}{l}\text { 9-El ambiente que hay en el hotel me hace sentir cómodo, } \\
\text { como en casa }\end{array}$ & 445 & 3.80 \\
\hline $\begin{array}{l}\text { 10-En las áreas comunes (alberca, playa, áreas de juegos) } \\
\text { hay un ambiente divertido y agradable }\end{array}$ & 445 & 3.71 \\
\hline
\end{tabular}

Fuente: Alzamora (2018).

En el área de Empatía sobresalen en el acuerdo positivo mayormente las siguientes afirmaciones:

- El personal del hotel siempre está atento a mis deseos y necesidades

- En el hotel me prestan una atención muy personal

- El personal del hotel siempre está con una sonrisa en el rostro; se muestra interesado por servir a los huéspedes.

\section{Dimensión: Fiabilidad}

Indicadores: Promesa, eficiencia y eficacia

Esta área evalúa la capacidad de desarrollar el servicio prometido seguramente y con precisión. La fiabilidad se evaluó con 4 preguntas, la cuales se presentan en el cuadro siguiente, y con los cálculos de su media por pregunta.

\section{Cuadro $\mathrm{N}^{\circ} 3$}

Fiabilidad

\begin{tabular}{|c|c|c|}
\hline & $\mathrm{N}$ & Media \\
\hline $\begin{array}{l}\text { 11-Si alguien en el hotel se compromete a hacer algo } \\
\text { por mí, lo hará }\end{array}$ & 445 & 3.85 \\
\hline $\begin{array}{l}\text { 12-Los distintos servicios que presta el hotel son } \\
\text { presentados correctamente desde la primera vez }\end{array}$ & 445 & 3.94 \\
\hline $\begin{array}{l}\text { 13-Cuando necesito ayuda o algo en particular, } \\
\text { siento toda la confianza de acudir al personal del } \\
\text { hotel para que se haga cargo de mi asunto }\end{array}$ & 445 & 4.12 \\
\hline
\end{tabular}




\begin{tabular}{|c|c|}
\hline $\begin{array}{l}\text { 14-En todo momento, a todas horas del día y en } 44 \\
\text { todos los lugares del hotel recibo un servicio de } \\
\text { calidad }\end{array}$ & 3.66 \\
\hline
\end{tabular}

Fuente: Alzamora (2018).

En el área de Fiabilidad sobresalen en el acuerdo positivo mayormente las siguientes preguntas:

- Cuando necesito ayuda o algo en particular, siento toda la confianza de acudir al personal del hotel para que se haga cargo de mi asunto

- Los distintos servicios que presta el hotel son presentados correctamente desde la primera vez

A continuación, se presentan los resultados de las preguntas 13 y 14 .

\section{Dimensión: Capacidad de respuesta \\ Indicadores: Cortesía y confianza}

Esta área evalúa la prontitud en ayudar a los clientes y proveer un servicio rápido. La capacidad de respuesta se evaluó con 4 preguntas, la cuales se presentan en el cuadro siguiente, y con los cálculos de sus medias y desviación estándar por pregunta.

\section{Cuadro $\mathbf{N}^{\circ} 4$}

\section{Capacidad de respuesta}

\begin{tabular}{l|l}
\multicolumn{1}{c|}{$\mathrm{N}$} & Media \\
\hline $\begin{array}{l}\text { 15-El personal del hotel siempre está dispuesto a } 445 \\
\text { atenderme y ayudarme }\end{array}$ & 3.67 \\
\hline $\begin{array}{l}\text { 16-Si se me presenta un problema o necesito } 445 \\
\text { ayuda, el personal del hotel me ayudará a resolverlo } \\
\text { inmediatamente }\end{array}$ & 3.49 \\
\hline $\begin{array}{l}\text { 17-Si solicito algo del personal del hotel, me } 445 \\
\text { informarán exactamente cuando me lo } \\
\text { proporcionarán, cumplirán con ello }\end{array}$ & 4.08 \\
$\begin{array}{l}\text { 18-Si requiero de algo especial que generalmente } 445 \\
\text { no se encuentra en el hotel, sé que me ayudarán a } \\
\text { conseguirlo }\end{array}$ & 4.07 \\
\hline
\end{tabular}

Fuente: Alzamora (2018).

En el área Capacidad de respuesta sobresalen en el acuerdo positivo mayormente las siguientes afirmaciones:

- Si solicito algo del personal del hotel, me informarán exactamente cuando me lo proporcionarán, cumplirán con ello

- Si requiero de algo especial que generalmente no se encuentra en el hotel, sé que me ayudarán a conseguirlo

A continuación, se presentan los resultados de las preguntas 17 y 18 . 


\section{Dimensión: Seguridad \\ Indicadores: Disposición y agilidad}

Esta área evalúa la cortesía de los empleados y su capacidad en inspirar confianza y certeza; Esta área se evalúa con 4 preguntas, la cuales se presentan en el cuadro siguiente, y con los cálculos de sus medias y desviaciones estándar por pregunta.

\section{Cuadro $\mathrm{N}^{\circ} 5$ Seguridad}

\begin{tabular}{ll|l}
\multicolumn{1}{c|}{$\mathrm{N}$} & Media \\
\hline $\begin{array}{l}\text { 19-Me siento seguro de dejar mis } 445 \\
\text { pertenecías en mi habitación }\end{array}$ & 3.77 \\
$\begin{array}{l}\text { 20-Confío en la integridad de las personas } 445 \\
\text { que trabajan en el hotel }\end{array}$ & 4.09 \\
$\begin{array}{l}\text { 21-Confío en que nunca entrará al hotel } 445 \\
\text { alguien que no esté autorizado para } \\
\text { hacerlo }\end{array}$ & 4.19 \\
$\begin{array}{l}\text { 22-Me siento tranquilo y seguro dentro } 445 \\
\text { del hotel }\end{array}$ & 3.28 \\
\hline
\end{tabular}

Fuente: Alzamora (2018).

En el área Seguridad sobresalen en el acuerdo positivo mayormente las siguientes afirmaciones:

- Confío en que nunca entrará al hotel alguien que no esté autorizado para hacerlo

- Confío en que nunca entrará al hotel alguien que no esté autorizado para hacerlo

A continuación, se presentan los resultados por cada una de las preguntas.

\section{Resultados del cuestionario aplicado a los colaboradores}

A continuación, se presentan los datos demográficos de los colaboradores de los hoteles.

En cuanto al género, un $58.5 \%$ son del sexo masculino, y un $41.5 \%$ son del sexo femenino. En cuanto a la edad, un 30.9\% tienen edades entre 51 a 65 años, un 30.2\% tienen más de 65 años. Un $18.2 \%$ tienen edades de 21 a 35 años, un 15.3\% tienen entre 36 a 50 años, y solo un 5.6\% tienen 20 o menos años. Esto indica que los colaboradores son personas maduras.

En cuanto al nivel educativo un $88.4 \%$ tienen estudios universitarios, un $10.5 \%$ tienen estudios básicos, y un 1.0\% tienen otros niveles educativos. En cuanto al país de origen, un 87.8\% son del país de Panamá, un 11.2\% son de Colombia, y un 1.0\% son de República Dominicana.

Sobre la ocupación, se observa que un $73.8 \%$ son personal del área de servicio, un $21.1 \%$ son personal de gerencia, y un $5.4 \%$ son personal de administración. Siempre en los hoteles, la mayor parte del personal son del área de servicio.

A continuación, se presenta la información sobre satisfacción laboral recogida a través del cuestionario impartido a los colaboradores de los hoteles que fueron 484 colaboradores, las preguntas corresponden a cada dimensión a medir y a los Ítems que se desarrollan por dimensión. 


\section{Dimensión: Motivación y reconocimiento}

Indicadores: Conocimiento, motivación, relaciones, comunicación y participación

Esta categoría nos aporta información relacionada con el nivel de identificación por parte de los trabajadores sobre el lugar que ocupa la empresa donde trabajan en su sector de referencia. También nos aporta información sobre el nivel de conocimiento por parte de los trabajadores de las funciones y responsabilidades de su puesto de trabajo, el nivel de cohesión y pertenencia a un grupo de trabajo.

\section{Cuadro $\mathrm{N}^{\circ} 6$}

Preguntas del área de motivación y el reconocimiento, sus medias y desviación estándar.

\begin{tabular}{|c|c|c|c|}
\hline & $\mathrm{N}$ & Media & $\begin{array}{l}\text { Desv. } \\
\text { Desviación }\end{array}$ \\
\hline 1-Conoce la historia y trayectoria del hotel. & 484 & 4.30 & .903 \\
\hline $\begin{array}{l}\text { 2-Sus funciones y responsabilidades están bien } \\
\text { definidas. }\end{array}$ & 484 & 3.98 & 1.019 \\
\hline $\begin{array}{l}\text { 3-Está motivado y le gusta el trabajo que } \\
\text { desempeña. }\end{array}$ & 484 & 4.17 & .947 \\
\hline $\begin{array}{l}\text { 4-El nombre del hotel y su posición en la } \\
\text { industria hotelera es gratificante para usted. }\end{array}$ & 484 & 4.18 & .816 \\
\hline $\begin{array}{l}\text { 5-Las condiciones salariales para usted son } \\
\text { distintas. }\end{array}$ & 484 & 3.91 & 1.072 \\
\hline $\begin{array}{l}\text { 6-La relación con sus compañeros de trabajo es } \\
\text { buena. }\end{array}$ & 484 & 4.15 & .968 \\
\hline $\begin{array}{l}\text { 7-Le resulta fácil expresar sus opiniones en su } \\
\text { grupo de trabajo }\end{array}$ & 484 & 3.87 & .939 \\
\hline 8-Se siente parte de un equipo de trabajo & 484 & 4.34 & .886 \\
\hline $\begin{array}{l}\text { 9-La comunicación interna dentro de su área } \\
\text { funciona correctamente }\end{array}$ & 484 & 4.44 & 4.097 \\
\hline $\begin{array}{l}\text { 10-Se siente participe de los éxitos y fracasos de } \\
\text { su área de trabajo }\end{array}$ & 484 & 4.20 & .952 \\
\hline
\end{tabular}

Fuente: Alzamora (2018).

En la categoría Motivación y Reconocimiento sobresalen en el acuerdo positivo mayormente las siguientes preguntas:

- La comunicación interna dentro su área funciona correctamente

- Se siente parte de un equipo de trabajo

- Conoce la historia y trayectoria del hotel.

- Se siente participe de los éxitos y fracasos de su área de trabajo

- Está motivado y le gusta el trabajo que desempeña. 


\section{Dimensión: Área y ambiente de trabajo \\ Indicadores: Organización y seguridad}

Esta área brinda información relacionada con las condiciones de trabajo relacionadas con la organización y seguridad, Este factor se evalúa con 3 preguntas. En el siguiente cuadro, vemos que la pregunta que, muestra mayores respuestas es la referente a que mantiene su lugar de trabajo limpio y libre de obstáculos.

\section{Cuadro $\mathrm{N}^{\circ} 7$}

Preguntas del área y ambiente de trabajo, sus medias y desviación estándar

\begin{tabular}{l|l|l}
\multicolumn{1}{c|}{$\mathrm{N}$} & Media & $\begin{array}{l}\text { Desv. } \\
\text { Desviación }\end{array}$ \\
\hline $\begin{array}{l}\text { 11-El trabajo de su área } 484 \\
\text { o línea está bien } \\
\text { organizado }\end{array}$ & 4.09 & .848 \\
\hline $\begin{array}{l}\text { 12-Las condiciones de } 484 \\
\text { trabajo de su línea son } \\
\text { seguras }\end{array}$ & 4.02 & .764 \\
\hline $\begin{array}{l}\text { 13-Mantiene su lugar de } 484 \\
\text { trabajo limpio y libre de } \\
\text { obstáculos }\end{array}$ & 4.26 & .772 \\
\hline
\end{tabular}

Fuente: Alzamora (2018).

\section{Dimensión: Formación e Información \\ Indicadores: Capacitación y desarrollo}

Esta área nos aporta información relacionada con los planes de formación que se aportan a los trabajadores, la formación básica impartida y las posibilidades de desarrollo profesional que se ofrece a los trabajadores. Esta área se evalúa con 3 preguntas. A continuación de muestras las medias de las respuestas. Se observa que la media es más alta en la pregunta que se refiere a que el hotel le proporciona la oportunidad para su desarrollo profesional (Media 4.00).

\section{Cuadro $\mathrm{N}^{\circ} 8$}

Preguntas del área de ambiente de trabajo, su medias y desviación estándar

\begin{tabular}{ll|l|l}
\multicolumn{1}{c|}{$\mathrm{N}$} & Media & $\begin{array}{l}\text { Desv. } \\
\text { Desviación }\end{array}$ \\
\hline $\begin{array}{l}\text { 14-Recibe la formación necesaria para } 484 \\
\text { desempeñar correctamente su trabajo. }\end{array}$ & 3.87 & 1.018 \\
\hline $\begin{array}{l}\text { 15-Cuando necesita formación } 484 \\
\text { específica, al margen de la establecida en } \\
\text { el plan de formación, ha sido satisfecha }\end{array}$ & 3.81 & .872 \\
$\begin{array}{l}\text { 16-El hotel le proporciona la } 478 \\
\text { oportunidad para su desarrollo } \\
\text { profesional }\end{array}$ & 4.00 & .949 \\
\hline
\end{tabular}

Fuente: Alzamora (2018) 


\section{Dimensión: Servicio al cliente}

Indicadores: Experiencia y compromiso

Esta área describe el juicio global del cliente a cerca de la excelencia o superioridad del servicio. El factor servicio al cliente se evalúa con 4 preguntas. La pregunta 18 y 19 mostraron medias altas, es decir que se preocupan por cumplir con las clientes y reciben frecuentemente capacitación en temas de servicio al cliente.

\section{Cuadro $\mathrm{N}^{\circ} 9$}

Preguntas del área de servicio al cliente, sus medias y desviación estándar

\begin{tabular}{l|l|l}
\multicolumn{1}{c|}{$\mathrm{N}$} & Media & $\begin{array}{l}\text { Desv. } \\
\text { Desviación }\end{array}$ \\
\hline $\begin{array}{l}\text { 17-Posee experiencia previa en temas de } 484 \\
\text { atención al cliente }\end{array}$ & 4.07 & .924 \\
\hline $\begin{array}{l}\text { 18-Se preocupa por cumplir con las } 484 \\
\text { necesidades de los clientes }\end{array}$ & 4.53 & .748 \\
\hline $\begin{array}{l}\text { 19-Recibe frecuentemente capacitación } 484 \\
\text { en temas de servicio al cliente }\end{array}$ & 4.10 & .997 \\
\hline $\begin{array}{l}\text { 20-Percibe de sus compañeros de trabajo } 484 \\
\text { compromiso en atender a los clientes }\end{array}$ & 4.04 & .970 \\
\hline
\end{tabular}

Fuente: Alzamora (2018).

\section{Discusión de resultados}

La industria hotelera panameña muestra un descenso en sus actividades, lo cual hace necesario mejorar el servicio al cliente, como una forma de atraer turistas, se ha señalado que es importante conocer el grado de satisfacción de los huéspedes, a través de la realización de encuestas de opinión, tal como se ha hecho en esta investigación.

Los clientes o huéspedes mostraron medias de respuestas más altas sobre Fiabilidad (3.91), seguido de Empatía (3.89), luego Confianza (3.86). Esto quiere decir que consideran que reciben un buen servicio del hotel, un trato personalizado, y sienten confianza en hospedarse en los hoteles analizados. Pero aún estos aspectos se pueden mejorar para pasar de ser buenos a ser excelentes, y esto se puede lograr a través de capacitaciones puntuales.

Los clientes evalúan positivamente la Capacidad de respuesta del personal y las instalaciones y las facilidades, pero existe la oportunidad de mejorar esos aspectos. Es necesario la optimización del servicio, como así también la fluidez de la comunicación entre los distintos departamentos, niveles jerárquicos y los clientes.

Los colaboradores muestras medias altas en el área de servicio al cliente (4.09), seguido por el área de Motivación y reconocimiento (4.07). Esto nos indica que es bueno el servicio al cliente y están motivados, pero existe una posibilidad de mejorar a través de capacitaciones y acciones en favor de los colaboradores. 
Los colaboradores opinan que existen un buen ambiente de trabajo, y que se debe mejorar los aspectos de Formación e Información. Consideran que se debe reforzar la cooperación, el profesionalismo y el trabajo en equipo, motivando al personal para lograr los objetivos previstos.

Los hoteles en Panamá cuentan con política o procedimiento en la busca de la satisfacción del cliente, pero la frecuencia de capacitación no es la adecuada. Es necesario divulgar y capacitar a todo el personal que el servicio al cliente se ha convertido al pasar de los años en motivo esencial en la decisión del cliente al escoger donde realizar la compra de algún producto y/o servicio en especial en las empresas en las cuales su naturaleza es la prestación de servicio, como la industria hotelera, todo esto da como resultado que el servicio actualmente sea un factor fundamental en la competitividad en los negocios.

\section{Bibliografía}

ALZAMORA, M.; HERRERA, V.; FONG, D.; PORTILLO, Y.; WONG, T.; BARUCO, K.; MELHADO, R.; RUIZ, G.; DEL CID, J.; JUSTINIANI, A.; CÓRDOBA, L.; ANDIÓN, S. y PAZMIÑO, E. (2014). Estudio de la percepción de los turistas sobre la calidad del servicio al cliente en la Ciudadde Panamá. Investigación y PensamientoCrítico. Universidad Santa María la Antigua (USMA). Volumen 2, Número 4 (Mayo-Agosto). Pp. 4-58. Disponible:

https://usma.ac.pa/public_html/wp-content/uploads/2018/01/investigacion-pensamiento-criticoAlzamora-cols.pdf

Autoridad de Turismo de Panamá (ATP) (2017). Boletín Estadístico 2017. Departamento de Estadística. Disponible:

http://www.atp.gob.pa/sites/default/files/documentos/resumen_ejecutivo_2017.pdf

Autoridad de Turismo de Panamá (ATP) (2018a). Resumen Estadístico Enero a Junio 2018. Departamento de Estadística.

Disponible:http://www.atp.gob.pa/sites/default/files/documentos/resumen_estadistico_de_enero _a_junio_2018.pdf

Autoridad de Turismo de Panamá (ATP) (2018b). Oferta de Alojamiento 2008-2017.

Departamento de Estadística.

Disponible:http://www.atp.gob.pa/sites/default/files/documentos/oferta_de_alojamiento.pdf

BENAVIDES, C. (2012). Calidad y Productividad en el Sector Hotelero Andaluz. Tesis

Doctoral. Universidad de Málaga. España.

COEXPHAL/EUROEMPLEO (2013). Cuestionario de Satisfacción Laboral. Servicio Andaluz de Empleo. Fondo Social Europeo. Disponible:

file:///C:/Users/amand/Downloads/Cuestionario $\% 20$ sobre $\% 20$ Satisfacci $\%$ C3\%B3n $\% 20$ Laboral$3 \% 20(3) \cdot \mathrm{pdf}$

FALCES C.; SIERRA, B.; BECERRA, A. \&BRIÑOL, P. (1999). HOTELQUAL: a scale for measuring perceived quality in lodging services. Estudios'Turísticos.Vol. 139. Pp 95- 110. 
GALLEGO, M.; y CASANUEVA, C. (2010). Dirección y organización de empresas turísticas. EdicionesPirámide. Madrid.

GETTY, J. \&THOMPSON, K. (1994). The relationship between Quality, Satisfaction and Recomending Behaviour in lodging decisions. Journal of Hospitality and Leisure Marketing, Vol. 2 (3).

HERNÁNDEZ, R.; FERNÁNDEZ, C. y BAPTISTA, M. (2014). Metodología de la Investigación. 6ta Edición. México.

MARTÍNEZ, M.; GUILLÉN, E.; BARBEITO, S. (2012). La calidad y su gestión: análisis y revisión teórica, Editorial Académica Española, Alemania.

MI DIARIO (2018). Revista Forbes destaca a Panamá como destino popular en 2018. Midiario.com, 29 de septiembre de 2018. Disponible: https://www.midiario.com/panama/nacionales/revista-forbesdestaca-panama-como-destino-popular-en-2018

Organización Mundial del Turismo OMT (UNWTO). (2015). Hotel Classification Systems: Recurrence of criteria in 4 and 5 star hotels. Disponible: http://prohotelia.com/wpcontent/uploads/2015/03/UNW'TO-Hotel-Classification-System.pdf

Organización Mundial del Turismo OMT (UNWTO). (2017a). UNWTO Annual Report 2017. Disponible:https://www.e-unwto.org/doi/pdf/10.18111/9789284419807

Organización Mundial del Turismo OMT (UNWTO). (2017b). Tourism and the Sustainable Development Goals - Journey to 2030. World Tourism Organization (UNWTO) and United Nations Development Program (UNDP). Disponible: https:/ /www.eunwto.org/doi/pdf/10.18111/9789284419401

Real Academia Española (RAE) (20016). Diccionario de la Lengua Española. Disponible: http://www.rae.es/recursos/diccionarios/drae

RUIZ, A.V., VÁZQUEZ, R. y DÍAZ, A.M. (1995): "La calidad percibida del servicio en establecimientos hoteleros de turismo rural". Papers de Turisme, No 19.

SPINELLI, M. \& CANAVOS, G. (2000). Investigating the relationship between employee satisfaction and guest satisfaction. Cornell hotel and restaurant administration quarterly, 41(6), 29-33.

TAMAYO, J. y VEGA, U. (2017). El Servicio al Cliente como Elemento Fundamental en la Industria Hotelera. Revista Turydes: Turismo y Desarrollo. No 23 (diciembre). Disponible: bttp:/ / www.eumed.net/ rev/turydes/23/ cliente-industria-botelera.btml

ULACIA, Z. (2015). Factores que Inciden en la Calidad de la Experiencia Alojativa en Hoteles de Ciudad 4 y 5 Estrellas en la Habana. Tesis Doctoral. Universidad de La Habana. 
VILLANUEVA, J. (2017). Turismo como motor del crecimiento económico sostenible: el caso especial del turismo rural. Tesis Doctoral. Universidad de Castilla-La Mancha. Disponible: https://ruidera.uclm.es/xmlui/bitstream/handle/10578/16465/TESIS $\% 20$ Villanueva \%20\%C3\%81 lvaro.pdf?sequence $=1$

World Economic Forum (WEF) (2017). The Travel \& Tourism Competitiveness Report 2017. Geneva.

World Travel \& Tourism Council (WTTC) (2018). Travel \& Tourism Economic Impact 2018 World. Disponible: https://www.wttc.org/-/media/files/reports/economic-impactresearch/regions-2018/world2018.pdf

ZEITHAML, V.; PARASURAMAN, A. \& BERRY, L. (1990). Delivering Quality Service. The Free Press, New York.

ZEITHAML, V.; BERRY, L. \& PARASURAMAN, P. (1990). Delivering Quality Service: Balancing Customer Perceptions and Expectaitions. Free Press. New York.

ZEITHAML, V.; BERRY, L.\& PARASURAMAN, A. (1993). The nature and determinants of customer expectations of service. Journal of the Academy Marketing of Science 21(1), 1-23

ZEITHAML, V. y BITNER, M. (2002). Marketing de servicios. Editorial McGraw-Hill interamericana. 2da. Ed. México. 